\title{
Outbreaks and the management of 'second-order friction': Repurposing materials and data from the health care and food systems for public health surveillance
}

\author{
Angie M. Boyce \\ Center for Population and Development Studies, Harvard T.H. Chan School of Public Health, USA \\ aboyce@hsph.harvard.edu
}

\begin{abstract}
In the US, the public health system plays a key role in identifying unsafe food in the food supply. This identification work (public health surveillance) entails piecing together and reworking materials and data from the health care and food sectors to identify the ultimate cause of the problem. As such, the public health system depends heavily on infrastructures built for other purposes to achieve its goals. Using the case of foodborne outbreak detection, this article enhances the ethnographic analysis of second-order systems by incorporating the concepts of 'repurposing' and 'friction' to analyze this dependent relationship, the challenges it entails, and the broader sociopolitical and ethical consequences of connecting heterogeneous infrastructures. I examine how actors within the secondorder system of public health conduct the practical work of repurposing materials and data from other sectors, and grapple with the inescapable presence of 'second-order friction' between their system and infrastructures built to achieve other goals.
\end{abstract}

Keywords: second-order friction, repurposing, foodborne disease outbreak surveillance

\section{Introduction}

Food supply chains are highly complex. They rely upon a large and diffuse network of interdependent infrastructures to get food from farm to fork, and align producer supply with consumer demand. If food becomes contaminated somewhere along the way, often the problem only gets discerned much after the fact, when an outbreak occurs. The visible signal that something has gone wrong is when end consumers get sick and seek medical care. However, the job of the health care system is to treat patients, and so it focuses its efforts on fulfilling that function. In the US, going as far as identifying the food that made the consumers sick is not part of its responsibility, and so the health care system only collects information relevant to fulfilling its clinical function. Figuring out what food caused the problem is the responsibility of the public health system, which 
must piece together and rework materials and data from the health care and food systems to identify the ultimate source of the outbreak, and help ensure the safety of the food supply.

To be efficacious, outbreak surveillance depends on robust linkages between the disparate sectors of public health, health care, and food production. Scholars have theorized systems that depend heavily on infrastructures built for other purposes as 'second-order systems' (Braun \& Joerges, 1994; Van der Vleuten, 2004). Second-order systems refer to "the process of networking parts of different first-order systems for specific, macro-level social domains" (Braun \& Joerges, 1994: 27). Braun and Joerges developed the concept in their study of a European organ transplantation network. This cross-national network depended on the interlinking of a variety of existing infrastructures ('first-order systems'), such as road and air transportation, telephony, long-distance data transmission, and hospitals, to achieve the goal of getting organs from donors to recipients in an expedient fashion. The concept of a second-order system has been used to look at the macro-level structural/functional aspects of these kinds of systems and how they develop over time. Complex adaptive systems scholars have studied the interdependencies of infrastructures using modeling and simulation to understand system vulnerabilities and the implications of rare or extreme events (Rinaldi et al., 2001). What is not addressed in these research traditions are two important aspects to infrastructural interdependency: the daily practical work of actors who create and maintain dependent systems, and the sociopolitical and ethical consequences of connecting heterogeneous infrastructures.

This article enhances the ethnographic analysis of second-order systems, bringing both practical work and its broader consequences to the foreground by introducing additional analytic language for understanding multi-infrastructural dynamics. As Vertesi (2014) argues, we need more vocabulary for understanding how actors skillfully work to bring multiple infrastructures into alignment, and work around given constraints and limitations. Using the concepts of 'repurposing' and 'friction', this article helps surface some of the 'invisible work' (Star, 1999) involved in making infrastructures built for other purposes serve public health needs, and connect that invisible work to its larger sociopolitical and ethical consequences.

Second-order systems rely heavily on infrastructures built for other uses, and actors within those systems encounter multiple challenges in their work because of their system's dependent relationship. I argue that the concepts of 'repurposing' and 'friction' help us more deeply understand this dependent relationship and the challenges it entails. 'Repurposing' is the adaptation of things that were created for one purpose to be used in a different way. As actors within second-order systems conduct the practical work of repurposing materials and data from other systems, they grapple with the inescapable presence of 'second-order friction,' or resistive force, between their system and infrastructures that were built to achieve other goals.

Surfacing the invisible work of repurposing and its sociopolitical and ethical dimensions is especially important for understanding public health. Public health practitioners often lament that public health is invisible when it works, because people are not getting sick. This invisibility contributes to the neglect of public health infrastructure, and ultimately, the health of populations. Using the concepts of repurposing and second-order friction, I tack back and forth between the practical work of maintaining second-order systems, and the sociopolitical and ethical consequences of that work, as a form of 'infrastructural inversion,' to better appreciate the "depths of interdependence of technical networks and standards on the one hand and the real work of politics and knowledge production on the other" (Bowker \& Star, 2000: 34). This article describes and explains how public health actors responsible for foodborne outbreak surveillance repurpose materials and data from the health care and food sectors to achieve system goals.

I draw empirical material from a larger threeyear project that examined the evolution of foodborne outbreak surveillance systems from a historical and ethnographic perspective. That project used a 'strategically-situated' approach, sampling key sites within the larger distributed system where participants were themselves 
managing questions of scale and distribution (Geiger \& Ribes, 2011). My understanding of the system's broad contours came from six months of organizational ethnography at the Centers for Disease Control and Prevention (CDC), and four months of regulatory work at the Food and Drug Administration (FDA). My look at the system from a more local perspective draws largely from a week-long site visit at a state public health department and laboratory that involved observation of work practices and interviews with staff. It was there that I was able to get a closer look at how public health practitioners managed the daily work of repurposing materials and data, and the frictions they worked to overcome. To deepen the analysis, I also draw selectively from other data sources, including one-on-one interviews with state and federal public health and regulatory scientists, policy documents, field notes from scientific meetings, and relevant scientific articles and media stories. For data analysis and theory construction, I used an abductive analysis process to seek a "situational fit" between my ethnographic observations of outbreak-related work and STS theory (Timmermans \& Tavory, 2012). Abductive analysis led me to the large technical systems and knowledge infrastructures literatures, which informed the development of the secondorder friction concept.

Because my approach is strategically-situated, it is limited by its partial view. It emphasizes the vantage point of the second-order system, which is built and operated by public health scientists. Observing the interactions between the system and existing infrastructures from other locales would reflect different facets of repurposing and friction, highlighting instead the concerns of the builders and operators of other infrastructures. Another limitation of the study is that I looked at only one state public health department and laboratory, when there is significant variation between states (e.g. funding, population size, degree of centralization). I do not capture differences between states in this study. However, the examples of repurposing and friction I discuss reflect more general challenges faced by many public health practitioners in this second-order system.
The remainder of the article proceeds as follows. In the next section, I develop a conceptual framework and situate it in the STS literature. After that I examine second-order friction from a broad, structural vantage point. Then I take a more local look at the practical work of repurposing and actors' management of frictions in day-today public health surveillance. Before concluding the article, I look at the friction associated with second-order adaptation to change in other infrastructures.

\section{Conceptual framework and literature review}

The concept of a second-order system comes out of the large technical system field inspired by Hughes (1983, 1987). Since Braun \& Joerges' (1994) pioneering study, Van der Vleuten (2003) has built upon the second-order system idea through an analysis of the Dutch food supply chain. Secondorder systems have three overarching properties. They are:

1. Parasitic. Second-order systems opportunistically borrow from other systems and infrastructures to achieve their goals, and tend to maintain less of their own substance. There are several advantages to a parasitic structure. It may be too costly, infeasible, or even impossible to create a contained system with dedicated infrastructure. The latter is true for both organ transplantation and disease outbreaks, in that they both involve unpredictable accidents at their source. Since donors or illnesses can come from anywhere at any time, second-order system builders must mobilize existing infrastructures to support their efforts.

2. Reliant on databases. Though second-order systems may maintain less of their own substance, they still require dedicated infrastructure. Databases often serve as key components of second-order systems, because they are powerful tools for coordinating spatially distributed, multi-scalar, temporally-complex phenomena. They help provide foci for drawing natural, social, and digital orderings together (Hine, 2006). 
3. Dependent. While there are many advantages to second-order systems' parasitic structure, there can be significant disadvantages. Because they draw substance from heterogeneous infrastructures, they tend to be less "insulated" from malfunctions and changes in other systems (Braun \& Joerges, 1994). Second-order system builders have multiple aspects of their system that are not under their control, resulting in an 'asymmetrical dependence' (Van der Vleuten, 2003; Mayntz, 1993). This is why questions of power are so relevant to understanding the relationships between second-order systems and existing infrastructures.

The second-order system concept is an analytic tool that helps analysts examine systems reliant on the interconnection of multiple infrastructures. In my view the concept is best applied to situations where readily identifiable second-order system builders actively work to mobilize multiple infrastructures in service of achieving a clearly defined goal. Navigating any complex contemporary environment involves working in and between systems and with multiple infrastructures, and produces "fleeting moments of alignment," but not necessarily a stable whole (Vertesi, 2014). By contrast, second-order systems must regularize alignment to achieve system goals. This is a challenge because absolute stability is not possible because of a parasitic structure with many disparate elements not under second-order system builders' control. As such, second-order systems are best seen as unstable wholes, made stable enough by the practical work of actors committed to keeping disparate infrastructures aligned.

Given the key role that the practical work of actors plays in holding second-order systems together, it is important to develop conceptual ways of describing and analyzing this work. The concept of repurposing is a particularly good one to analyze the work of holding second-order systems together, because it calls attention to the original and new purpose and context of development, as well as their interaction effects. The general idea of repurposing has a long history in STS, but only recently have scholars refined it into a more specific analytic concept.
That different social groups can have different uses and meanings for the same objects and artefacts is a well-established STS principle. The Social Construction of Technology (SCOT) approach (Pinch \& Bijker, 1984; Kline \& Pinch, 1996) emphasized that users were an important social group playing a role in technology construction, and highlighted the interpretive flexibility around the different meanings different social groups ascribed to a technology. Star \& Griesemer's (1989) influential concept of the boundary object captured how actors in different but intersecting social worlds could coordinate cooperative work without coming to consensus. Domestication theory (Lie \& Sørensen, 1996; Silverstone \& Hirsch, 2003) called attention to the integration of technologies in everyday life, involving the reshaping of technologies and user meanings and identities in the process. Dourish (2003) called the process of adopting, adapting, and incorporating technologies into working practices 'appropriation, seeking to discover features of technical design that could fruitfully support it. Data 'reuse,' or data that was collected for one purpose being used to study a new problem, is an analogous phenomenon (Zimmerman, 2008).

In contrast to earlier work that focused on individual technological artifacts or a single system, scholars have more recently elaborated the concept of repurposing to better appreciate the dynamic relationships between heterogeneous infrastructures and multiple systems. Jarzabkowski \& Pinch (2013) have called for a focus on repurposing as one of the key concepts to advance further empirical studies of sociomateriality. They argue that prior studies have tended to focus on affordances and new functions and intentions for objects. Instead, scholars should examine the situated activities actors accomplish in repurposing objects in context, and the social interactions between groups involved in purposing and repurposing things. Jackson (2014) has pointed to activities like repurposing as important phenomena to investigate understudied technological dimensions such as repair, maintenance, breakdown, and decay, instead of the more attention-getting novelty, growth, and progress. Repurposing offers us new ways to think about innovation and inequalities involved 
in social relationships around technologies. Ribes \& Polk (2015) highlight the importance of repurposing to maintain long-term research endeavors and enable the investigation of new research objects. Importantly, they point out, repurposing requires effort to ensure that infrastructure can facilitate research on new objects of investigation without disruption.

When considering the dynamic relationships between different infrastructures and different social groups involved in aligning and repurposing materials and data, it is important to have ways of talking about how actors enact and experience those relationships. Tsing (2005:4) theorizes friction as the "awkward, unequal, unstable, and creative qualities of interconnection across difference." It is the force resisting the motion of moving surfaces in contact, and requires significant energy and effort to overcome. Tsing uses friction to tack back and forth between scales, interrogating the production of the global and local, universal and particular, and the constitutive relationships between them. Also, she finds that encounters across difference do not just entail challenges for those involved, but can also stimulate creative possibilities and the development of new cultural forms.

Edwards (2010) and Edwards et al. (2011) have taken a typological perspective to theorizing friction in knowledge infrastructures, to explore the challenges associated with different aspects of knowledge production. Actors in knowledge infrastructures must commit time, energy, attention, and resources to overcome many resistive forces. What the concept of friction usefully does in this arena is to emphasize the materiality of information, which is often framed as immaterial. 'Data friction' results when data must move between people, substrates, organizations, or machines, and in the work required to collect, check, store, move, receive, and access it. 'Computational friction' is associated with the work required to process data and turn it into information and knowledge. 'Metadata friction' arises with the work involved in managing and communicating information about data, important for making it shareable in multi-disciplinary, collaborative efforts. 'Science friction' refers to the challenges encountered by different scientific disciplines when they work together on related problems.

This article tracks the 'second-order friction' that results when actors in the second-order system repurpose materials and data from other systems and infrastructures. In that repurposing work, they encounter frictions of many forms. To understand foodborne disease outbreak surveillance, it is useful to consider second-order friction at multiple scales, tacking back and forth between the broad structural aspects of connecting health care, public health, and food production systems, and the intricate work of repurposing materials and data at a more local level. It is also important to connect the practical work of repurposing to its broader social context. Indeed, managing daily second-order frictions can make the political and ethical consequences of foodborne outbreak surveillance seem distant to the actors doing it. Yet those political and ethical consequences are what provide the work with larger meaning and moral purpose-making the food supply safer. As one interviewee told me during my site visit, "It can get hard to 'see the forest for the trees' in day-to-day surveillance. We take a 'fire engine' response to problems, but we can lose the bigger picture."1 The interviewee used the forest-trees idiomatic expression to emphasize how difficult public health workers found it to discern broader patterns when overwhelmed by the details of their daily work. In addition, the emergencyresponse 'fire engine' mode of work presented another challenge in feeling connected to the work's broader political and ethical implications.

\section{Second-order friction from the forest}

The specific second-order system examined here is a foodborne outbreak surveillance system. However, I emphasize that it is layered in multiple ways. At the micro-layer, the system can be defined in terms of specific tools, such as the surveillance database. At the meso-layer, it is important to consider how foodborne outbreak surveillance is housed within the larger public health system, so that issues such as state-federal relationships come into play. At the macro-layer, a broad system goal for outbreak surveillance is to 
network the first-order systems of health care and food production, to identify the ultimate cause of food contamination.

Foodborne outbreak surveillance is one of many types of public health surveillance, which Thacker \& Berkelman (1988) define as the ongoing systematic collection, analysis, and interpretation of health data, integrated with data dissemination and application to programs for prevention and control. This epidemiologic monitoring of patterns of disease at a population level is a function typically conducted by government entities, who are responsible for official disease reporting. In the US context, the idea of disease reporting dates back to the $18^{\text {th }}$ century and concerns about potential epidemics of communicable diseases such as cholera, yellow fever, and smallpox. The national system in place today began to take shape in the last few decades of the $19^{\text {th }}$ century, growing out of late $18^{\text {th }}$ century developments, such as sanitary reform movements and the rise of municipal and state boards of health as governing bodies (Fairchild et al., 2007; Koo \& Wetterhall, 1996; Duffy, 1990).

Because government bodies are responsible for producing official statistics about the health of the nation, significant resources and effort must be put into transforming data from disparate sources not originally intended for public health use, into trustworthy numbers that have public health meaning. Public health surveillance systems often repurpose data from a variety of sources, such as "clinical laboratory test results, patient encounter data, environmental monitoring, pharmaceutical sales data, insurance claims data, vaccination registries, vital statistics, morbidity and mortality data, and notifiable disease reports" (Mirhaji, 2009). One of the primary sources of data for many public health surveillance systems is the health care sector. The parasitic relationship of the public health sector to the health care sector is formalized by disease reporting laws made by state legislatures, which require health care professionals to report diseases of public health concern to the government.

By their own rights, the American public health and health care systems are each large, complex, fragmented, and highly regulated, which makes the task of connecting the two all the more chal- lenging. One of the most significant sources of friction in the public health system is the balance of power in America's federalist system. Some states have given local (county and city) governments little authority to govern public health, while other states (deemed "home rule") give local authorities more control. While states are the primary entity responsible for health in the public sector, the federal government is responsible for coordinating the dissemination of knowledge and policy-making, priority-setting, and providing technical assistance and resources for strengthening state and local capacity (Institute of Medicine, 1988). An outgrowth of this complex distribution of power is a complex distribution of responsibility for collecting and managing data and materials.

The structure of the health care system causes friction for patients and caregivers. They must navigate a pluralistic delivery system comprised of large numbers of small providers in different kinds of venues, such as primary care facilities, specialty clinics, and diagnostic centers, and in any one facility, a single patient may be cared for by a physician, nurse, pharmacists, medical assistant, or other caregiver (Bodenheimer, 2008). In this context, a patient's medical record is not a simple object that can easily travel. The medical record is a complex infrastructural entity comprised of numerous written and digital traces mediating the production of the patient's body, the hospital as an organization, and wider connections to multiple bodies politic (Berg \& Bowker, 1997). Though many clinical environments make use of information systems to produce electronic medical records more efficiently, it is important to understand that the data are produced and used in the context of direct clinical care. Even repurposing data for in-unit clinical process improvement presents a number of challenges (Morrison et al., 2013), let alone repurposing this data for public health use.

To touch on the making of the medical record in the foodborne context, whether an illness is a foodborne one or not is unclear at the outset, when a person first gets sick and seeks medical care. Both the clinic and the laboratory are involved in evaluating individuals that present symptoms of gastroenteritis. Clinicians perform 
differential diagnosis by analyzing various aspects of the patient's symptoms. For example, timing of the onset of symptoms suggest infection from different pathogens (Donnenberg \& Narayanan, 2013). If a person gets sick within 6 hours of ingesting a problem food, Staphylococcus aureus is more likely to be the cause. If onset of symptoms is between 6 and 48 hours, some of the possible causes are Shigella, Salmonella, or Escherichia coli.

Making sense of different clinical symptoms can help direct the laboratory to perform the right kinds of tests that will identify the infectious agent from a patient's biological sample (normally stool). The particular clinical diagnostic test that can be repurposed by the public health system is the stool culture, which involves taking a stool sample from a patient, and placing it in a special medium that encourages particular kinds of bacteria to grow, based on different phenotypic characteristics of different organisms. After the bacterial cells are grown in a selective medium ("cultured"), laboratory technicians segregate the pathogen of interest; that is, they separate a pure variety of a single pathogen from other bacteria. This step is always necessary because in the human body and in the broader environment, microbes exist in multi-member communities. The segregated pathogen is commonly referred to as an "isolate."

Second-order friction is associated with getting and repurposing the isolate and clinical data from both the clinic and the laboratory to the public health system. Clinical data and laboratory materials and data are often reported through separate channels and at different times. Public health practitioners must winnow down clinical data to repurpose it, for instrumental and legal reasons. From an instrumental perspective, only a small subset of data from the larger medical record is of interest for public health surveillance (for example, a patient's name, address, phone number, date of birth, gender, diagnosis, and symptom onset date). From a legal perspective, particular precaution must be taken in the handling of patient "protected health information" (PHI), governed by the Health Insurance Portability and Accountability Act (HIPAA), federal regulation to protect the privacy and confidentiality of individuals' medical records. Since counties and states are primary entities responsible for health in the public health system, fuller patient records containing $\mathrm{PHI}$ tend to be housed at the state level, and only linked to the federal level through de-identified codes.

Clinical data must be winnowed down, but clinical laboratory data are not sufficient for informing outbreak detection, and must be deepened for public health use. For outbreak detection, it is not enough to know whether the bacteria is a Shigella or Staphylococcus aureus. The isolate must be sent to public health laboratories to generate more specific data about its type, so that public health officials can determine whether an outbreak has occurred. Since the mid-1990s, state public health laboratories have been performing standardized molecular subtyping on foodborne isolates using a DNA fingerprinting method, pulsed-field gel electrophoresis (PFGE). When bacteria share PFGE fingerprints, this suggests that they might be members of the same outbreak, and initiates an investigation.

To manage the frictions of coordinating heterogeneous materials and data in foodborne disease outbreak surveillance, databases are key tools in the second-order system. On the laboratory side, from the early 2000s onward, state public health laboratories have been contributing PFGE data in PulseNet, a network and database for early-warning foodborne outbreak detection coordinated by the CDC (Swaminathan et al., 2001; Tauxe, 2006). PulseNet relies on commercial software for analysis of biological data, with customized scripts for data entry, queries, and submission of data to the national level (GernerSmidt et al., 2006). To facilitate collaboration and data sharing around national outbreak investigations, the OutbreakNet epidemiologic network of federal, state, and local public health officials uses off-the-shelf web-based platforms to support their investigative work (MacDonald, 2012).

While repurposing a patient's medical record and isolate entails connecting the health care system to the public health system, to help identify the contaminated food that is the source of an outbreak entails connecting the public health system to the food system. The food system is inordinately complex; Sobal et al. (1998) have conceptualized it as the "food and nutrition system" to account for its various subsystems 
(producer, consumer, and nutrition) and multiple stages (production, processing, distribution, acquisition, preparation, consumption, digestion, transport, and metabolism.) In contrast to the relatively more stable linkage between clinical laboratories and public health laboratories required for isolate shipping, the connections between the public health system and the food system are made in the context of specific outbreak investigations on an ad-hoc basis. However, what is stable is a key mediating actor between the public health system and the food system-state food safety regulatory agencies. Across states, food safety regulatory authorities can alternatively reside in departments of agriculture, food protection, and/or environmental health (Council to Improve Foodborne Outbreak Response, 2009).

Foodborne outbreak investigations rely on a method of epidemiologic case interviewing to collect "food histories" from sickened individuals. Once the health care system reports patient data to the public health system, public health staff contact cases to ask them questions about the foods they consumed in their homes, in the homes of friends and family, in restaurants and other food establishments, within the relevant disease incubation period, or time between exposure and illness (MacDonald, 2012). Who conducts food history interviewing varies significantly by state. In some states, particularly those under home rule, food history interviews are conducted by county public health nurses, while in others they are conducted by epidemiologists in county or state departments of public health. These interviews help generate public health practitioners generate hypotheses about the potential foods that may have caused a case's illness.

To figure out which food caused the outbreak, more evidence from the food supply and distribution chain must be collected to legally implicate it so it can be removed from interstate commerce and stop making people sick. Regulatory officials have jurisdiction over commercial data. They use a methodology called 'traceback' to figure out how food moved through the supply and distribution chain by collecting food records (National Environmental Health Association, n.d.). They begin at the endpoint of consumption, the transaction between a point of service and the consumer, and obtain distribution records to identify shipments and suppliers back through the chain until a common source is found, an outcome they call convergence. Officials seek data from cases and points of service such as purchase date and location, brands and descriptions of food items, packaging and labeling information, and lot numbers. They also ask those points of service for lists of suppliers, delivery information, shipping documents such as invoices and bills of lading, and inventory records. Ultimately, officials aim to find the common source at the production or farm-level, to identify what caused the contamination issue. Supply chains are designed to get food from producers to consumers, so in repurposing food records to trace a food's journey from consumer to producer, regulatory officials encounter significant second-order friction.

\section{Second-order friction from the trees}

"Outbreaks have been pushing communication and community," a technician observed. From this technician and many of the other public health scientists I interviewed, I repeatedly heard an emphasis of the importance of relationshipbuilding across distributed organizations. Collegiality was an important element smoothing the friction of coordinating work and sharing objects and data between heterogeneous and dispersed groups. Previously, the technician stated, before the frequent detection of outbreaks, "state laboratory people didn't meet their epis and ag [state department of agriculture]." Since its inception, a significant amount of PFGE data has been accumulated in the PulseNet database, resulting in the increasingly frequent detection of possible outbreaks; in this laboratory technician's state alone, the state public health laboratory subtypes between 900-1,100 Salmonella isolates a year.

At first much of the coordination and communication were organized by phone calls and e-mails, but eventually the two groups decided that the epidemiologists should also have access to the PulseNet database, normally a tool managed by the laboratory. This repurposing of a national laboratory database as a local laboratory-epidemiology communication tool helped to facilitate better coordination between the two teams. More specifically, it helped the epidemiologists 
keep closer tabs on incoming information about isolates of interest.

However, giving the epidemiologists access to the PulseNet database did not entail a complete repurposing of the tool as a space for conducting epidemiologic data analysis. During the site visit, much of the daily work I observed foodborne epidemiologists conducting was in a database I call EDSS, for Electronic Disease Surveillance System. EDSS was a shared resource across the whole department of public health since it was a cross-disease database system for all reportable diseases. I found out that as a shared resource, it was a site of friction resulting from tensions between the surveillance needs for different diseases. A technical limitation of EDSS was that it was a standardized product created by a commercial vendor, and as such, the data fields it contained had to be common across disease domains. Customization was expensive, an epidemiologist informed me; "every change is dollars."2

Besides generic data fields, another challenge was that it was difficult to extract data from EDSS. The epidemiologist continued, "The system was built for putting in data, but in our line of work, we want it out. How do we search it?" Even though EDSS was ostensibly built for putting in data, observing the friction associated with this process revealed the human work required to repurpose data from the health care system. Several times a week, an epidemiologist would update EDSS with foodborne case data. Updating EDSS involved an epidemiologist checking a shared team e-mail account for new notifications of laboratory reports that were sent from clinical laboratories who had identified reportable pathogens in patient stool samples. These laboratory reports came as a PDF attachment of a standardized form including limited demographic information about the patient and their laboratory diagnosis (e.g. specimen collected, type, results). The epidemiologist created a new "incident ID number" for the patient in EDSS, and typed the patient's clinical and laboratory diagnostic data into the matching fields.

To make up for the fact that customization could not be built into EDSS and address additional foodborne-specific needs for extracting and analyzing data, the foodborne epidemiology team created a local database housed in a folder on a shared drive. The aim of the local database was to tie the clinical laboratory data together with the data that would be coming in later from the state public health laboratory. Though clinical laboratories sent laboratory report forms to the foodborne epidemiology team in the state department of public health once their diagnostic results were in, the clinical laboratories also shipped the isolate to the state public health laboratory to perform PFGE.

As I spoke with the epidemiologic team about extracting and analyzing data from EDSS, I learned that their local database helped serve as an accountability mechanism, to monitor the status of isolates and keep track of important information generated at different times by different players. Outbreaks are time-pressured health emergencies, where delays result in more people getting sick. Many delays reflect the friction between the second-order public health system and the first-order health care system. The local database, an epidemiologist pointed out, helped the team make sure that "we are getting all the information we need." 3

The daily work of extracting and analyzing EDSS data began with the epidemiologist opening the local database, and creating new entries for the case data. A particularly important data field for this step was labeled "resolution status." Under resolution status, the epidemiologist chose to identify the cases as "suspect." The term suspect meant that the clinical laboratory had submitted a report with clinical diagnostic information to the department of public health, and likely (but not definitively) shipped the isolate to the state public health laboratory, but the state public health laboratory had not yet conducted its confirmatory testing on the isolate. When the state public health laboratory finished its testing, it would e-mail a laboratory report to the public health department with the test results. From this laboratory report, the epidemiologist would enter the testing results, as well as the isolate's laboratory ID number, marrying the epidemiologic case data with the laboratory isolate data. By marking the resolution status as suspect, it would be possible to monitor whether the other distributed entities in the chain of work had completed their tasks. 
That EDSS had not been customized to support disease-specific needs was a significant source of friction for its users in their work to extract and analyze data, and many of the epidemiologists I spoke with would have liked to be part of the design decision-making. In a tight and precarious fiscal environment, building customization into design of the database was not prioritized. However, while EDSS certainly could have been designed differently to better support diseasespecific needs, another aspect to this story is that multiple databases are often used to support different kinds of work, perspectives, and priorities (Bietz \& Lee, 2009). The local database reflected the importance of getting information about isolates generated by others at different times, and linking the isolate data back to the clinical case data to enable outbreak surveillance.

\section{Adapting to first-order change}

While the local database attests to the crucial role that isolates play in foodborne outbreak surveillance, and helps play a role in creating accountability for isolates, it is not a tool that can compel data from heterogeneous infrastructures to come together. Reflecting on the power dynamics involved in second-order/first-order infrastructural relations, in a Working Group report, the Association of Public Health Laboratories (2014) emphasized that only some aspects of isolate "turnaround time" were within the control of public health laboratories:

\footnotetext{
"When assessing laboratory testing turn-aroundtimes for foodborne illness specimens, the steps from specimen collection to final results can be generally divided into those 'within our control' and those 'beyond our control'. The steps up through clinical laboratory analysis and submission to the PHL are often times beyond PHL control; however, there are ways in which public health can encourage and influence rapid and thorough submission of clinical isolates."
}

An outgrowth of America's patchworked federalist system is that states vary significantly in their legal mandates for disease reporting. While many states require reporting of case data, where clinicians and clinical laboratories are obligated to report diagnoses of notifiable diseases and limited patient data to local or state public health authorities, fewer states mandate clinical laboratories to conduct isolate reporting and ship isolates to public health laboratories.

In fact, the state I visited did not mandate isolate submission. Mandating the shipment of isolates would have made their consignment a part of clinical laboratory infrastructure. However, since clinical laboratories did not "own" this responsibility, second-order system builders at the federal level used the strategy of providing grant funding to incentivize clinical laboratories to submit isolates to the state public health laboratory. The grant money provided shipping containers to clinical laboratories, and paid for a specialized courier service to transport isolates from clinical laboratories to the state public health laboratory. What the grant money did not cover was the time and labor of the technicians in clinical laboratories to pack isolates in the subsidized shipping containers, and mail them to the public health laboratory. The constraints of this "soft money" program reflect the more general problem of fragmented and precarious funding for public health infrastructure (Baker et al., 2005).

When I toured the state public health laboratory, I saw evidence of major second-order friction threatening the interoperability of the health care and public health systems in outbreak surveillance. Near the laboratory entrance, several mundane items on a cart caught my eye. I was not surprised to see isolates fixed in agar slants and petri dishes, but I was surprised to see that the cart held orange-capped jars of stool specimens. I asked the laboratory technician why the stool specimens were there, because I had assumed that the clinical laboratories were always responsible for isolating bacteria from stool specimens. He clarified that this was not a frequent practice, but that the clinical laboratory who sent the samples had performed diagnostic testing on them using a "rapid" test, and had not isolated the pathogen from the stool. So the state public health laboratory asked the clinical laboratory to forward the stool samples on, so that it could isolate the bacteria for public health surveillance. ${ }^{4}$

The repurposing of isolates from the health care system has been a key element of the second- 
order system of foodborne outbreak surveillance, especially in the post-1990s era of molecular detection. However, the science and technology of microbial identification has not remained static, with increased development and uptake of culture-independent diagnostic testing (CIDT) in clinical laboratories. CIDT means that diagnostic results can be produced without needing to isolate organisms from samples, which would sever the key connection between the health care and public health systems that enables public health surveillance. CIDT methods offer several advantages over culture-based methods to clinical laboratories, such as more rapid test results, cheaper per specimen costs, shorter turnaround times, and lower complexity (Atkinson et al., 2013). Quicker and cheaper diagnoses can mean improved, more cost-efficient clinical care for patients.

However, some public health scientists have wondered whether these changes have heralded the opening of "Pandora's box," reducing the ability for both health care and public health surveillance systems to diagnose and identify diseases at the individual and population-levels (Janda \& Abbott, 2014). As a second-order system, public health surveillance is particularly vulnerable to changes in the health care system. If CIDT methods are adopted before public health surveillance systems can adapt to the displacement of isolates, it will harm public health surveillance capacities like national foodborne outbreak detection, as well as the tracking of specific trends in infections (Cronquist et al., 2012). Cronquist et al. (2012) see the isolate dilemma as a difference in the values of the clinical and public health systems, in how the systems differently define what constitutes a "good" diagnostic test. There are shared values between sectors (accuracy, rapidity, cost), but also many differences in values and how those values are prioritized. Clinicians are oriented to treating individual patients, emphasize speed over accuracy, and typically need less detailed information about isolates. Public health practitioners are focused on the health of populations, may emphasize accuracy over speed, and typically need more detailed information about isolates.

One interviewee offered his reflections on this problem, identifying both economic and moral aspects to the friction around CIDT:
Interviewee: The outside force, the commercial sector, wants to sell the latest and greatest test to laboratories that don't require a live organism... They are not interested in surveillance, they are interested in making money. It is a factor difficult to control.

Interviewer: But is this [CIDT] meeting the needs of hospitals and consumers?

Interviewee: It's hard to divorce what the hospital wants versus what the company wants. New generation products justify themselves by saving time. They are making inroads into laboratories, everybody wants the latest and best methods. Companies give laboratories machines for free, and then charge for the tests. They know laboratories can't afford the machines, but if the laboratories buy the assay for the next umpteen years... If the commercial sector was altruistic they wouldn't introduce these tests. There's no status quo, nothing's "good enough."

Attesting to the second-order character of public health surveillance, the scientist framed the commercial sector as an "outside force" that the public health sector had difficulty controlling. He argued that the commercial sector was using a razor-and-blade type business model by giving away a platform and making money on buyers' subsequent dependency on the assays. The broader literature on the CIDT problem emphasizes the importance of stakeholder collaboration around finding solutions. But in the context of an interview, one scientist took a more pointed view, even raising questions about morality, "altruism," and when a test should be seen as "good enough."

As the operators of public health surveillance systems work to understand and ultimately solve the isolate dilemma, and try to maintain connections between the health care system and the public health system, they engage in creating expectations about the future. Expectations about the future are generative and dynamic, guiding present activities, defining roles, clarifying duties, fostering investments, and shaping strategies for leveraging opportunities and facing risks (Borup et al., 2006). In April 2012, several public health groups convened an expert consultation on CIDT, and "brainstormed potential solutions to address the anticipated impacts" of CIDT on public 
health surveillance (Association of Public Health Laboratories, 2012). During the consultation, one official stated that state public health laboratories were beginning to become the "primary entities" culturing specimens, rather than clinical laboratories.

The technician I spoke with in the state public health laboratory informed me that, on a smallscale, conducting isolation of bacteria from specimens in the public health laboratory was not an insurmountable problem. With their traditional microbiology training, staff in the public health laboratory had the technical know-how to perform isolations. However, to accomplish this shift at a large-scale, he surmised, more trained staff and funding would be required in public health laboratories. Increasing the amount of staff trained in traditional microbiology as well as building up laboratory infrastructure for traditional culturing did not necessarily make long-term sense, he pointed out. A major paradigm shift faced the public health system, which would involve the displacement of traditional microbiology and shift to computationally-intensive genomic and bioinformatic infrastructure.

The adoption of CIDT in the health care system is a change that threatens the connection between the health care and public health systems so crucial for outbreak surveillance. However, changes in other systems do not always result in risks to interoperability. Second-order systems can be adaptable, and changes in other infrastructures can result in improvements to secondorder systems. This can be seen in the dynamics around a food system innovation. In the 1990s, supermarkets created computerized card-based programs to offer promotions to shoppers, as well as to collect and store individual purchase transaction data in computerized databases, a practice growing out of decades of marketing techniques employed to encourage shopper loyalty and increase consumer spending (Bellizzi \& Bristol, 2004). As transaction data has accumulated, these databases have become "sophisticated competitive weapons" for electronic marketing, expanding beyond their initial function as a consumer discount delivery mechanism (Hammel, 1996).

Public health surveillance has been increasingly repurposing shopper card data to assist in outbreak investigations, and this method helps to address some of the limitations of traditional food history interviews. Understandably, consumers have difficulty recounting in detail every food item that they ate, especially if much time has elapsed, or if they are still feeling sick, or experience anxiety while being interviewed by a public health official (Mann, 1981). Seeing this phenomenon through the lens of repurposing adds further analytic insight. In everyday life, food is what people consume for the purposes of nourishment and enjoyment. The food history interview is a method for repurposing the food consumption practices of people as data for public health surveillance, and the (understandable) fallibility of human memory reflects the friction involved in this repurposing.

Several factors have enabled the second-order system to not just adapt to this change in the food system, but to help improve outbreak detection because of it. First, regulatory officials across federal and state levels are playing a more active role during outbreak investigations. Second, a data access process has been negotiated to manage friction associated with a complex set of laws. Laws governing the disclosure of shopper card data vary significantly across states, but in order to get shopper card data from commercial entities, public health officials usually must request shopper card numbers from individual cases and ask them to sign a consent form. Companies are not necessarily required to share information, but often times do so on a cooperative basis. Third, the second-order system has significant data analysis capability because of the central role that epidemiologic expertise plays in it. The frictions associated with incorporating shopper card data have more to do with the morality and materiality of repurposing than making sense of the data.

The second-order repurposing of supermarket card data for public health surveillance raises an interesting kind of moral friction illuminated by Nissenbaum's (2009) work on the politics of privacy surrounding different surveillance systems. Shopper cards are one of many examples of "dataveillance" in modern society, where people's dayto-day activities, interactions, and transactions can be tracked through information (Clarke, 1988). An important aspect to dataveillance is that infor- 
mation may be used for purposes unintended in the original design of tracking systems. In health care, Nissenbaum (2009: 6) notes, surveillance devices are lauded "hallmarks of high-quality care", while other surveillance activities, such as shopper loyalty card programs, are often criticized for infringing consumer privacy as retailers mine data for marketing insights. Public discourse on the repurposing of shopper card data for public health use reflects the moral friction surrounding dataveillance. In a media article (Vitals, 2013), one state public health official emphasized public health's careful safeguarding of information and limited use of data for illness prevention purposes, as well as performing professional boundary work (Abbott, 1988; Gieryn, 1983) between different government entities. The article quoted, "We are the government, but we aren't that part of the government... we're the good guys."

A great deal of material friction lies in the repurposing of materials and data from the food system, where public health workers must engage with the materiality of food records, and of food itself. Officials must process shopper card data in whatever form is given to them by companies, a form shaped by the companies' internal systems and the way that those systems export data. And though the repurposing of shopper card data has helped address some of the limitations of food history data, both only contribute to identifying possible food exposures. Only by tracing foods' journeys from the point of consumption back through distribution and production can investigators determine what food caused the problem and where it came from. My interviews with regulatory officials provided a picture of the frictions they encountered while repurposing food record data during traceback investigations. One state regulatory official shared how her department had to create an entire "war room" dedicated to a major traceback investigation. ${ }^{6}$ Hand-drawn traceback diagrams on butcher paper covered the walls, and binders of paper records from suppliers FedExed to the agency filled the tables. The official used these examples to underscore how manual, time-consuming, and stressful the traceback process was for the staff charged with processing such heterogeneous data in the time-pressured context of an outbreak investigation.
Traceback involved a high amount of friction not only because each commercial entity created its own records for its own purposes in varying formats (paper and electronic), but that food itself did not remain a stable object in the process of traveling through the supply chain. Another regulatory official illustrated this issue by describing the traceback of a tomato moving through the supply chain. The whole tomato, she said, ripened from green to red in transit, and as it ripened, each color would transform how the tomato was categorized at different points in the chain of distribution, for example, entering in one place as a "vine-ripe," exiting as a "greenhouse," and recorded at retail as a "red round bulk."7 Another official pointed out that not only did categories for tomatoes change, but the quantities and forms in which tomatoes were packaged also shifted throughout the supply chain. ${ }^{8}$ He described how, once tomatoes go from fields to packinghouses, middlemen take them out of the boxes they were shipped in because tomatoes ripen at different rates. Tomatoes from multiple fields and farms are shipped to the same packinghouse. The packinghouse regrades and resorts the tomatoes, and may reuse the original shipping boxes, but place tomatoes that came from different fields and farms into those original boxes. Such practices of sorting tomatoes, he argued, were not reflected in the paper records. Significant second-order friction arose in managing the differences in the material production and distribution of food, record-keeping practices for tracking those processes, and repurposing those food records for public health use.

\section{Conclusions}

Second-order friction inevitably arises in systems that depend heavily on other infrastructures to achieve system goals. Adding the analytic vocabulary of repurposing and friction to the study of second-order systems helps to foreground the important role that the practical, skillful, and often times invisible work of actors within them plays in maintaining the connections between heterogeneous infrastructures. The concepts of repurposing and friction also help analysts connect this 
daily maintenance work to its larger sociopolitical and ethical consequences.

From a broader structural perspective, connecting the health care, public health, and food sectors is a difficult endeavor because each of these systems in their own rights is large, complex and distributed, with an array of different institutional actors. Two key objects that are repurposed from the health care system are the medical record and the isolate, so that relevant clinical and laboratory data can be woven together to inform outbreak detection. Second-order system actors encounter much friction in this repurposing work, whether they are winnowing clinical data, safeguarding protected health information through a layered relationship between the state and federal levels, or getting isolates to and through the public health system.

To manage that daily friction, multiple databases served as key tools. Faced with the constraints of a non-customized, cross-disease surveillance system built for the primary purpose of data input, actors in the state public health department created local databases as workarounds. In addition to databases, collegiality mattered immensely for smoothing secondorder friction. As an object of joint responsibility, outbreaks served as a driver of relationshipbuilding across distributed organizations.

Changes in other infrastructures typically pose threats to second-order systems. The displacement of culture-based methods in the health care system in favor of culture-independent rapid tests fits that general pattern. However, that public health surveillance could incorporate the change in the food system to create shopper card databases demonstrates that second-order systems can be adaptable. Additionally, building relationships between second-order systems and other infrastructures involves not just technical considerations, but political and moral ones. The problem of culture-independent testing demonstrates how much power the health care system has in defining what constitutes health, through its control over how materials from patients are transformed into data, for what purposes, and in which forms. The example of mobilizing shopper cards for public health surveillance highlights the moral frictions that can surround the repurposing of materials and data, as the traces of everyday life are turned into information that spurs action in the world.

The analysis of second-order friction in the repurposing of materials and data should be pursued in additional case studies. More work is needed to understand how second-order systems can adapt to changes in other infrastructures, as well as situations in which second-order systems hold power over other infrastructures. In particular, global health is a prime arena to investigate these dynamics. Some global health surveillance programs have disrupted fragile health care systems in resource-poor countries, marginalizing the building of local health care infrastructure in favor of attracting investment in top-down surveillance systems and categorical disease initiatives (Calain, 2007). The 2013-2014 Ebola epidemic in West Africa is urging some in the global health field to shift focus from addressing specific diseases to strengthening weak health care infrastructure (Barbiero, 2014).

Indeed, one of the biggest ongoing controversies in global public health surveillance involves friction over the repurposing of biological materials in disease reporting. In 2006, the Indonesian government stopped contributing H5N1 virus samples to the global influenza surveillance network run by the World Health Organization (WHO). Indonesia protested the WHO sharing virus samples with Western pharmaceutical companies, who used the samples to create new $\mathrm{H} 5 \mathrm{~N} 1$ vaccines, arguing that the prices for the vaccines charged by pharmaceutical companies were unaffordable. Though benefit-sharing policies have been put in place to make vaccine manufacture and distribution more equitable, innovations in the synthesis of viruses from genetic sequencing data may obviate the need to directly repurpose virus samples, a dynamic akin to the culture-independent problem discussed above. These benefit-sharing policies do not apply to genetic sequencing data since they do not define genetic sequencing data as "biological material" (Gostin et al., 2014). Conflicts can arise not just around how data are generated and moved, but because of differences in how "data" are defined and valued (Levin, 2014). Further analyses of second-order friction will help illumi- 
nate the serious moral and political implications of purposing and repurposing "materials," "data," and building interconnections between disparate systems and infrastructures.

\section{Notes}

1 Interview conducted 6/4/2013.

2 Interview conducted 6/3/2013.

3 Interview conducted 6/3/2013.

4 Field notes, 6/5/2013.

5 Interview conducted 8/16/12.

6 Interview conducted 11/19/13.

7 Interview conducted 8/2/2013.

8 Interview conducted 12/16/13. 


\section{References}

Abbott AD (1988) The System of Professions: An Essay on the Division of Expert Labor. Chicago: University of Chicago Press.

Association of Public Health Laboratories (2012) Culture-Independent Diagnostics Forum: Charting a Path for Public Health. Available at: http://www.aphl.org/aphlprograms/food/CIDT/Documents/FS_2012Apr25_ CID-Forum-Summary.pdf (accessed 22.1.2016).

Association of Public Health Laboratories (2014) Rapid and Thorough Submission of Clinical Specimens/ Isolates to Public Health Laboratories. Available at: http://www.aphl.org/aphlprograms/food/laboratoryaccrediation/Documents/2014Jan_Clinical-Isolates-Work-Group-Report.pdf (accessed 22.1.2016).

Atkinson R, Maguire H, Gerner-Smidt, P (2013) A Challenge and an Opportunity to Improve Patient Management and Public Health Surveillance for Food-Borne Infections through Culture-Independent Diagnostics. Journal of Clinical Microbiology 51 (8): 2479-2482.

Baker Jr EL, Potter MA, Jones DL, Mercer, SL, Cioffi JP, Green LW, Halverson PK, Lichtveld MY, Fleming DW (2005) The Public Health Infrastructure and Our Nation's Health. Annual Review of Public Health 26: 303-318.

Barbiero, VK (2014) It's not Ebola ... it's the systems. Global Health: Science and Practice, 2(4): 374-375.

Bellizzi JA, Bristol T (2004) An Assessment of Supermarket Loyalty Cards in One Major US Market. Journal of Consumer Marketing 21(2): 144-154.

Berg M, Bowker G (1997) The Multiple Bodies of the Medical Record. The Sociological Quarterly 38(3) 513-537.

Bietz MJ, Lee CP (2009) Collaboration in Metagenomics: Sequence Databases and the Organization of Scientific Work. In: Proceedings of the 11th European Conference on Computer Supported Cooperative Work, Vienna, Austria, 7-11 September 2009: 243-262). Springer: London.

Bodenheimer T (2008) Coordinating Care: A Perilous Journey Through the Health Care System. New England Journal of Medicine 358(10): 1064-1071.

Borup M, Brown N, Konrad K, Van Lente H (2006) The Sociology of Expectations in Science and Technology. Technology Analysis \& Strategic Management 18(3-4): 285-298.

Bowker GC \& Star SL (2000) Sorting Things Out: Classification and its Consequences. Cambridge: MIT Press.

Braun I \& Joerges B (1994). How to Recombine Large Technical Systems: The Case of European Organ Transplantation. In: Summerton J (ed) Changing Large Technical Systems. Boulder: Westview Press, 25-51.

Calain P (2007) From the Field Side of the Binoculars: A Different View on Global Public Health Surveillance. Health Policy and Planning 22(1): 13-20.

Clarke R (1988) Information Technology and Dataveillance. Communications of the Association for Computing Machinery 31(5): 498-512.

Council to Improve Foodborne Outbreak Response (2009) Guidelines for Foodborne Disease Outbreak Response. Atlanta: Council of State and Territorial Epidemiologists. Available at: http://www.cifor.us/ documents/CIFOR\%20Industry\%20Guidelines/CIFOR-Industry-Guideline.pdf (accessed 22.1.2016).

Cronquist AB, Mody RK, Atkinson R, Besser J, D'Angelo MT, Hurd S, Robinson T, Nicholson C \& Mahon BE (2012) Impacts of Culture-Independent Diagnostic Practices on Public Health Surveillance for Bacterial Enteric Pathogens. Clinical Infectious Diseases 54 (Suppl 5): S432-S439.

Donnenberg MS \& Narayanan S (2013) How to Diagnose a Foodborne Illness. Infectious Disease Clinics of North America 27(3): 535-554.

Dourish P (2003) The Appropriation of Interactive Technologies: Some Lessons From Placeless Documents. Computer Supported Cooperative Work 12(4): 465-490.

Duffy J (1990) The Sanitarians: A History of American Public Health. Urbana: University of Illinois Press. 
Edwards PN (2010) A Vast Machine: Computer Models, Climate Data, and the Politics of Global Warming. Cambridge, MA: MIT Press.

Edwards PN, Mayernik MS, Batcheller A, Bowker G \& Borgman C (2011) Science Friction: Data, Metadata, and Collaboration. Social Studies of Science 41(5): 667-690.

Fairchild AL, Bayer R \& Colgrove J (2007) Searching Eyes: Privacy, the State, and Disease Surveillance in America. Oakland: University of California Press.

Geiger RS \& Ribes D (2011) Trace Ethnography: Following Coordination through Documentary Practices. In: Proceedings of the 2011 44th Hawaii International Conference on System Sciences: 1-10. IEEE Computer Society.

Gerner-Smidt P, Hise K, Kincaid J, Hunter S, Rolando S, Hyytiä-Trees E, Ribot EM \& Swaminathan B (2006) PulseNet USA: a five-year update. Foodbourne Pathogens \& Disease 3(1): 9-19.

Gieryn TF (1983) Boundary-Work and the Demarcation of Science From Non-Science: Strains and Interests in Professional Ideologies of Scientists. American Sociological Review 48(6): 781-795.

Gostin LO, Phelan A, Stoto MA, Kraemer J \& Reddy KS (2014) Virus Sharing, Genetic Sequencing, and Global Health Security. Science 345(6202): 1295-1296.

Hammel F (1996) Data Base Dividends. Supermaket Business 51(3): 109-117.

Hine C (2006) Databases as Scientific Instruments and their Role in the Ordering of Scientific Work. Social Studies of Science 36(2): 269-298.

Hughes TP (1983) Networks of Power: Electrification in Western Society, 1880-1930. Baltimore: Johns Hopkins University Press.

Hughes TP (1987) The Evolution of Large Technological Systems. In: Bijker W \& Pinch TJ (eds) The Social Construction of Technological Systems. Cambridge: MIT Press, 51-82.

Institute of Medicine Committee for the Study of the Future of Public Health (1988) The Future of Public Health 88(2). Washington DC: National Academy Press.

Jackson SJ (2014) Rethinking Repair. In: Gillespie T, Boczkowski PJ \& Foot KA. Media technologies: Essays on communication, materiality, and society. Cambridge: MIT Press.

Janda JM \& Abbott SA (2014) Culture-Independent Diagnostic Testing: Have We Opened Pandora's Box for Good? Diagnostic Microbiology and Infectious Disease 80(3): 171-176.

Jarzabkowski P \& Pinch T (2013) Sociomateriality is 'the New Black': Accomplishing Repurposing, Reinscripting and Repairing in Context. M@ n@gement 16(5):579-592.

Kline R \& Pinch T (1996) Users as Agents of Technological Change: The Social Construction of the Automobile in the Rural United States. Technology and Culture 37(4): 763-795.

Koo D \& Wetterhall SF (1996) History and Current Status of the National Notifiable Diseases Surveillance System. Journal of Public Health Management and Practice 2(4): 4-10.

Levin N (2014) What's Being Translated in Translational Research? Making and Making Sense of Data between the Laboratory and the Clinic. TECNOSCIENZA: Italian Journal of Science \& Technology Studies 5(1): 91-114.

Lie M \& Sørensen KH (1996) Making Technology Our Own?: Domesticating Technology Into Everyday Life. Oslo: Scandinavian University Press.

MacDonald P (2012) Methods in Field Epidemiology. Burlington: Jones \& Bartlett Learning.

Mann JM (1981) A Prospective Study of Response Error in Food History Questionnaires: Implications for Foodborne Outbreak Investigation. American Journal of Public Health 71(12): 1362-1366.

Mayntz R (1993) Policy-Netzwerke Und Die Logik Von Verhandlungssystemen. In: Adrienne Heritier. PolicyAnalyse. Kritik und Neuorientierung, Politische Vierteljahresschrift, Special Issue 24. Opladen. 
Mirhaji P (2009) Public Health Surveillance Meets Translational Informatics: A Desiderata. Journal of the Association for Laboratory Automation 14(3): 157-170.

Morrison C, Jones M, Jones R \& Vuylsteke A (2013) 'You Can't Just Hit a Button': an Ethnographic Study of Strategies to Repurpose Data from Advanced Clinical Information Systems for Clinical Process Improvement. BMC Medicine 11(1): 1-8.

National Environmental Health Association (n.d.) Traceback Investigations. Available at: http://www.neha. org/epi_ready/pdf/Foodborne_Disease_Resource_Materials/Information_Collected_for_Traceback_ Investigations_(Supplemental).pdf (accessed 22.1.2016).

Nissenbaum H (2009). Privacy in Context: Technology, Policy, and the Integrity of Social Life. Stanford: Stanford University Press.

Pinch TJ \& Bijker WE (1984) The Social Construction of Facts and Artefacts: Or How the Sociology of Science and the Sociology of Technology Might Benefit Each Other. Social Studies of Science 14(3): 399-441.

Ribes D \& Polk JB (2015) Organizing for Ontological Change: The Kernel of an AIDS Research Infrastructure. Social Studies of Science 45(2): 214-241.

Rinaldi SM, Peerenboom JP \& Kelly TK (2001). Identifying, Understanding, and Analyzing Critical Infrastructure Interdependencies. Control Systems, IEEE, 21(6): 11-25.

Silverstone R \& Hirsch E (2003) Consuming Technologies: Media and Information in Domestic Spaces. London: Routledge.

Sobal J, Khan LK \& Bisogni C (1998) A Conceptual Model of the Food and Nutrition System. Social Science \& Medicine 47(7): 853-863.

Star SL (1999) The Ethnography of Infrastructure. American Behavioral Scientist 43(3): 377-391.

Star SL \& Griesemer JR (1989) Institutional Ecology, Translations and Boundary Objects: Amateurs and Professionals in Berkeley's Museum of Vertebrate Zoology, 1907-39. Social Studies of Science 19(3): 387-420.

Swaminathan B, Barrett TJ, Hunter SB, Tauxe RV \& Force C (2001) PulseNet: The Molecular Subtyping Network for Foodborne Bacterial Disease Surveillance, United States. Emerging Infectious Diseases 7(3): 382-389.

Tauxe RV (2006) Molecular Subtyping and the Transformation of Public Health. Foodbourne Pathogens \& Disease 3(1): 4-8.

Thacker SB \& Berkelman RL (1988) Public Health Surveillance in the United States. Epidemiologic Reviews 10: 164-190.

Timmermans S \& Tavory, I (2012) Theory construction in qualitative research from grounded theory to abductive analysis. Sociological Theory 30(3): 167-186.

Tsing AL (2005) Friction: An Ethnography of Global Connection. Princeton: Princeton University Press.

Van der Vleuten E (2003) In Search of the Networked Nation: Transforming Technology, Society and Nature in the Netherlands During the Twentieth Century. European Review of History 10(1): 59-78.

Van der Vleuten E (2004) Infrastructures and Societal Change. A View from the Large Technical Systems Field. Technology Analysis \& Strategic Management 16(3): 395-414.

Vertesi J (2014) Seamful Spaces: Heterogeneous Infrastructures in Interaction. Science, Technology \& Human Values 39(2): 264-284.

Vitals NBC News (2013) Shopper Cards May Save Your Life, Food Safety Sleuths Say. 13 March. Available at: http://vitals.nbcnews.com/_news/2013/03/13/17273535-shopper-cards-may-save-your-life-food-safetysleuths-say?lite (accessed 1.7.2015).

Zimmerman AS (2008) New Knowledge From Old Data the Role of Standards in the Sharing and Reuse of Ecological Data. Science, Technology \& Human Values 33(5): 631-652. 